\title{
GIS Based Site Specific Major Nutrient Maps and Recommendations for Coconut (Cocus nucifera L.) Gardens
}

\author{
M. Dinesh Kumar ${ }^{1 *}$, Binny Gopal ${ }^{1}$ and B.C. Dhananjaya ${ }^{2}$ \\ ${ }^{1}$ Department of Agronomy, College of Agriculture, University of Agricultural and \\ Horticultural Sciences, Shivamogga- 577225, Karnataka, India \\ ${ }^{2}$ Department of Soil Science and Agricultural Chemistry, College of Agriculture, University of \\ Agricultural and Horticultural Sciences, Shivamogga- 577225, Karnataka, India \\ *Corresponding author
}

\begin{tabular}{|l|}
\hline Ke y w or d s \\
Coconut, GIS, Grid, \\
$\begin{array}{l}\text { Fertilizers, Site } \\
\text { specific, STV }\end{array}$ \\
\hline Article Info \\
\hline $\begin{array}{l}\text { Accepted: } \\
\text { 26 April } 2018 \\
\text { Available Online: } \\
\text { 10 May } 2018\end{array}$ \\
\hline
\end{tabular}

\section{Introduction}

The coconut palm (Cocos nucifera Linn.), is a member of the family Palmae (palm family). In the world, it occupies an area of 12196 thousand hectares with 69836 million nuts (Anon., 2016). Indonesia, Philippines and
India are the top three countries producing coconut. In India, it is grown mostly along the coastal regions in almost 18 states and three union territories with an area of 2089 thousand hectares, an annual production of 1427 milion nuts with the productivity of 22167 nuts per hectare (Anon., 2016). Karnataka stands third 
in the country regarding coconut production (5128 million nuts) and occupies sixth position productivity-wise. Major coconut growing districts in Karnataka are Tumkur, Hassan, Dakshina Kannada, Chikkamagaluru and Chitradurga, which together account for more than 85 per cent of the coconut-growing area. Hence, it is very important horticultural crop having a very high impact on the economy of farmers of Hill, Southern Transition and Central Dry Zones of Karnataka. By and large, the crop is being grown with middle to less care. The perennial nature of the crop, the tall upper canopy, unavailability of skilled labours, biotic stress, etc paves the way for less caring situations. The crop is grown in diversified soil and climatic conditions. The soils also differ with that of agro-climatic situations and not possible to generalize the soil nutrient status as it is a dynamic factor. The phyto availability of nutrient elements is highly dictated by different soil parameters such as nutrient concentrations, $\mathrm{pH}$, cation exchange capacity, organic carbon, etc., apart from types and varieties of the crop with their age (Kabata-Pendias, 2004). Meanwhile, Hartemink (2005) stated that large quantities of nutrients are immobilized in the above and below ground of perennial crops paving the way for lesser efficiency.

At present, there is a uniform recommendation of major nutrients to this crop across soils of various agro-climatic zones. The present recommendation for coconut is 330:200:800 $\mathrm{g}$ nitrogen, phosphorus and potassium per palm in two split dosages for all nut-bearing trees. By this crop or location is not justified as an excess or imbalanced application of nutrients not only wastes this limited, costly resource but also pollutes the environment and hinders the uptake of other essential nutrients. Thus exacting soil fertility management is of paramount importance not only for sustained growth and yield but also for its better use efficiency. An approach towards justifying such concerns is site specific nutrient management wherein spatial variations are considered for exacting fertilizer applications. For the purpose, well established yielding coconut gardens were selected across agroclimatic situations with an objective to classify the soil nutrient status and to understand the field variability. Spatial information is meaningfully synchronized using geographical information system for drawing fertility maps and recommendations were developed for improving the garden fertility status to obtain sustainable yields.

\section{Materials and Methods}

The study area included Chikkapattanagere of Chikkamagaluru district where tank fed irrigation is dominant, Doddaghatta of Davangere district enjoy the supply of tank irrigation around the year, Siriyur of Shivamogga district also enjoys the Bhadhra river water supply and Madhure of Chitradurga district with limited success of tube wells irrigation (Fig. 1). A total of 195 acres representing 99 farmers was chosen for the study belongs to Karnataka state, India. The details of the study sites are given in Table 1. The gardens selected were aged 2025 years with varying management levels. The standard grid technique of $50 \times 50 \mathrm{~m}$ was employed to draw soil samples from the selected study area at $0-30$ and $30-60 \mathrm{~cm}$ depth. Each grid is recognized by its GPS coordinate to help for geo-referencing of the study area.

Further, using GPS coordinates, the base maps of the study area was developed in GIS environment. Soil samples collected from each grid was dried, powdered, sieved with $2 \mathrm{~mm}$ mesh and analyzed by following the standard methods. For available nitrogen, the procedure of alkaline permanganate method of Subbaiah and Asija (1956) was employed. By following 
Brays and Olsen method (Jackson, 1973), available phosphorus was estimated. Potassium was extracted by shaking the requisite amount of soil sample with $1 \mathrm{~N} \mathrm{NH}$ OAc solution and estimated as outlined by Jackson (1973). Nutrient Index Values (NIV) were calculated using the following formula

$\mathrm{NIV}=\left[\left(\mathrm{PH}^{*} 3\right)+(\mathrm{PM} * 2)+(\mathrm{PL} * 1)\right] / 100$

Where, PH, PM and PL are the percentages of soil sample falling in the category of high, medium and low status given weighting factor of 3, 2 and 1 respectively (Ramamurthy and Bajaj, 1967). Utilizing soil analytical results for nitrogen, phosphorus and potassium on grid basis, maps of these nutrients were prepared based on low, medium and high classification criteria (Tandon, 1993). The points having the same category were grouped into class as a polygon by using inverse distant weighed method interpolation technique. Maps for the individual nutrients were generated using GIS. Based on these variable values of nutrients, by adopting soil test values (STV), fertilizers quantity for each grid was calculated. The package recommendation and the site specific recommendation based on STV approach are compared. For each location depending on its site specific variations, nutrient application approaches are advised for farmers.

\section{Results and Discussion}

The location wise variation of major nutrient contents is presented in Table 2 and the results are indicated in the following paragraphs.

Chikkapattangere: - The location is a shadow area just beside hilly zone, but tanks nearby filled due to assured rains of hilly delta during June to October (kharif) and the collected water sustain perennial crops during off rainy season. These areas receive around $650 \mathrm{~mm}$ rainfall during kharif and soils are alkaline and basic in reaction. Due to the availability of water crops are being grown resulting depletion of soil fertility and also experience more of abiotic stress situations like the variation in temperature, relative humidity, sunshine hours, etc. Lower status of available nitrogen (maximum of 174 and $123 \mathrm{~kg} \mathrm{ha}^{-1}$ ) was recorded at different depths. Soils recorded a maximum of 119 and $82 \mathrm{~kg} \mathrm{ha}^{-1}$ respectively for phosphorus content at the top and below depths (Table 2). Out of the samples tested, 70 and 29 per cent soils are medium and high for phosphorus status at the top layer of 0-30 $\mathrm{cm}$ and remained unaffected at a deeper layer of $30-60 \mathrm{~cm}$. At top layer of $0-30 \mathrm{~cm}$, soils are medium to high status (66 and $34 \%$ respectively) for potassium content while at deeper layer medium status reduced to 47 per cent and sharing the rest proportion equally for low and high status. In these two depths, nitrogen remained very low (NIV of 1.00) while phosphorous became adequate (NIV 2.29 and 2.28) for rating. Soils recorded NIV of 2.66 and 2.00 for potassium indicating high to the adequate rating.

Doddaghatta: - The location is blessed with both Bhadra river canal and as well tank feeder canal and hence assured a source of irrigation. Kharif season receives $580 \mathrm{~mm}$ rainfall and during off season it is well supported by irrigation and hence the management level is also at higher side, The soil reaction was varying with 20 per cent of samples collected showed acidic reaction while 44 per cent remained neutral and rest of the samples showed basic reaction. Soils had a mean value of 157 and $179 \mathrm{~kg} \mathrm{~N} \mathrm{ha}^{-1}$ in different depths. Hence, the samples were rated low with low NIV rating. Soils recorded a maximum of 50 and $64 \mathrm{~kg} \mathrm{ha}^{-1}$ phosphorus content respectively for depths studied and $>95$ per cent observed to be medium status, hence gained an adequate rating of NIV with 1.99 and 2.04. Out of the samples analyzed, 69 and 64 per cent remained medium status for 
potassium at depths of $0-30$ and $30-60 \mathrm{~cm}$ while rest of the samples showed high status. These trends provided a steady state of stability with NIV values of 2.31 and 2.26 and rated as adequate.

Madhure: - The location is dry belt and the crop was sustained mainly by tube well irrigation. The annual rainfall is $520 \mathrm{~mm}$ and more of sunshine, temperature, hot climate prevails during summer. The functional ability of tube well depend on rainfall and most of them are yielding low water and accordingly the management is also in low order. In the absence of any irrigation sources, farmers opt bore wells extensively for irrigating perennial crops. The 60 per cent of soils is having alkalinity and basic in reaction while remaining once observed to be mainly neutral in reaction. The distribution of nitrogen over profiles had shown uniformity with low content due to inherent capacity and less retention. Larger variations are noticed for phosphorus and potassium status as dictated majorly by the capacity of supply to the tune of requirement, turnover of the residuals, remaining, etc. The mean available value of 71 and $68 \mathrm{~kg} \mathrm{ha}^{-1}$ in different grids indicating the status of $\mathrm{P}$ in top layer remained high (around $66 \%$ ) to medium (around 33\%) and the reverse trend is observed at $30-60 \mathrm{~cm}$ depth. The available mean potassium value was at 250 and $184 \mathrm{~kg} \mathrm{ha}^{-1}$ for depths studied in different grids (Table 2). Hence, at the top layer of $0-30 \mathrm{~cm}, 75$ per cent of the samples were rated medium and 17 per cent of them were higher leaving the remaining samples as low status. At a depth of $30-60 \mathrm{~cm}$, the proportion of medium and high status decreased to 71 and 4 per cent respectively paving the way for more low status. The fertility rating of very low (NIV of 1.00) for nitrogen, high to adequate (NIV of 2.66 and 2.25) for phosphorus and adequate to low (NIV of 2.10 and 1.79) for potassium was achieved in these soils, demanding careful attention of both nitrogen and phosphorus fertilizers.

Siriyur: - The location comes under transitional tract blessed with canal water facility. The annual rainfall is around $850 \mathrm{~mm}$ during Kharif season. During summer, the crop is benefitted by canal water with moderate level of management. About 88 per cent of the samples analyzed are being classified under acidic in reaction.

The nitrogen levels varied from 62 to $425 \mathrm{~kg}$ $\mathrm{ha}^{-1}$ in top layer while at $30-60 \mathrm{~cm}$ depth the values recorded were from $45-235 \mathrm{~kg} \mathrm{ha}^{-1}$. In top layer of $0-30 \mathrm{~cm}$, nitrogen has shown low (around $62 \%$ ) to medium (around $37 \%$ ) status and nullified at deeper layer with low status. Phosphorus was high (around $74 \%$ ) to medium (around $26 \%$ ) status for $0-30 \mathrm{~cm}$ depth, while at deeper layer remained largely medium (85\%). Potassium largely had medium status (around $80 \%$ ) at top layer as it moves to deeper sections it become almost equal proportion of medium and low status.

Table.1 Details of the study sites

\begin{tabular}{|c|c|c|c|c|}
\hline & Chikkapattanagere & Doddaghatta & Madhure & Siriyur \\
\hline Total No. of farmers & 37 & 7 & 24 & 31 \\
\hline No of samples & 160 & 160 & 160 & 144 \\
\hline Area (acre) & 50 & 50 & 45 & 50 \\
\hline pH range & $8.10-8.90$ & $6.01-8.19$ & $5.60-9.40$ & $4.52-7.64$ \\
\hline Soil Reaction & Basic & $\begin{array}{c}\text { Neutral to } \\
\text { basic }\end{array}$ & $\begin{array}{c}\text { Neutral to } \\
\text { basic }\end{array}$ & $\begin{array}{c}\text { Predominantly } \\
\text { Acidic }\end{array}$ \\
\hline
\end{tabular}


Table.2 Soil status of major nutrients for different coconut gardens

CHIKKAPATTANAGERE

DODDAGATTA

\begin{tabular}{|c|c|c|c|c|c|c|c|c|c|c|c|c|c|c|c|c|}
\hline & \multicolumn{4}{|c|}{$0-30 \mathrm{~cm}$} & \multicolumn{4}{|c|}{$30-60 \mathrm{~cm}$} & \multicolumn{4}{|c|}{$0-30 \mathrm{~cm}$} & \multicolumn{4}{|c|}{$30-60 \mathrm{~cm}$} \\
\hline & pH & $\underset{(\mathrm{kg} / \mathrm{ha})}{\mathrm{N}}$ & $\begin{array}{c}\mathrm{P}_{2} \mathrm{O}_{5} \\
(\mathrm{~kg} / \mathrm{ha})\end{array}$ & $\begin{array}{c}\mathrm{K}_{2} \mathrm{O} \\
(\mathrm{kg} / \mathrm{ha})\end{array}$ & pH & $\begin{array}{c}\mathbf{N} \\
(\mathrm{kg} / \mathrm{ha})\end{array}$ & $\begin{array}{c}\mathbf{P}_{2} \mathrm{O}_{5} \\
(\mathrm{~kg} / \mathrm{ha})\end{array}$ & $\begin{array}{c}\mathrm{K}_{2} \mathrm{O} \\
(\mathrm{kg} / \mathrm{ha})\end{array}$ & pH & $\begin{array}{c}\mathrm{N} \\
(\mathbf{k g} / \mathbf{h a})\end{array}$ & $\begin{array}{c}\mathbf{P}_{2} \mathrm{O}_{5} \\
(\mathrm{~kg} / \mathrm{ha})\end{array}$ & $\begin{array}{c}\mathrm{K}_{2} \mathrm{O} \\
(\mathrm{kg} / \mathrm{ha})\end{array}$ & pH & $\begin{array}{c}\mathbf{N} \\
(\mathrm{kg} / \mathrm{ha})\end{array}$ & $\begin{array}{c}\mathbf{P}_{2} \mathbf{O}_{5} \\
(\mathrm{~kg} / \mathrm{ha})\end{array}$ & $\begin{array}{c}\mathrm{K}_{2} \mathrm{O} \\
(\mathrm{kg} / \mathrm{ha})\end{array}$ \\
\hline Maximum & 8.80 & 173.60 & 118.70 & 552.00 & 8.90 & 123.20 & 82.00 & 271.80 & 8.19 & 156.80 & 50.31 & 600.80 & 7.96 & 179.20 & 63.91 & 585.60 \\
\hline Minimum & 8.10 & 22.40 & 20.70 & 150.12 & 8.10 & 11.20 & 14.70 & 102.00 & 6.07 & 78.4 & 18.88 & 142.80 & 6.01 & 72.80 & 23.74 & 81.60 \\
\hline Average & 8.46 & 97.71 & 70.62 & 297.47 & 8.50 & 77.87 & 45.02 & 164.27 & 7.06 & 123.17 & 35.92 & 290.99 & 7.07 & 116.26 & 37.48 & 267.47 \\
\hline Standard deviation & 0.35 & 23.76 & 15.84 & 18.03 & 0.28 & 1.71 & 1.51 & 23.76 & 0.54 & 18.18 & 7.72 & 94.93 & 0.56 & 23.14 & 7.34 & 106.60 \\
\hline Standard error & 0.98 & 19.42 & 13.28 & 61.74 & 0.99 & 13.78 & 9.17 & 30.40 & 0.06 & 2.04 & 0.88 & 10.75 & 0.06 & 2.62 & 0.83 & 12.07 \\
\hline \multirow[t]{3}{*}{$\begin{array}{c}\text { Ratings } \\
\text { of samples }\end{array}$} & Acidic $=9$ & $\begin{array}{l}\text { Low } \\
=80\end{array}$ & $\begin{array}{l}\text { Low } \\
=01\end{array}$ & $\begin{array}{l}\text { Low } \\
=00\end{array}$ & $\begin{array}{l}\text { Acidic } \\
=00\end{array}$ & $\begin{array}{l}\text { Low } \\
=80\end{array}$ & $\begin{array}{l}\text { Low } \\
=04\end{array}$ & $\begin{array}{l}\text { Low } \\
=21\end{array}$ & $\begin{array}{l}\text { Acidic } \\
=16\end{array}$ & $\begin{array}{l}\text { Low } \\
=78\end{array}$ & $\begin{array}{l}\text { Low } \\
=01\end{array}$ & $\begin{array}{l}\text { Low } \\
=00\end{array}$ & $\begin{array}{l}\text { Acidic } \\
=15\end{array}$ & $\begin{array}{l}\text { Low } \\
=78\end{array}$ & $\begin{array}{l}\text { Low } \\
=00\end{array}$ & $\begin{array}{l}\text { Low } \\
=04\end{array}$ \\
\hline & Neutral $=00$ & Medium $=00$ & Medium $=56$ & Medium $=53$ & $\begin{array}{l}\text { Neutra } \\
l=00\end{array}$ & $\begin{array}{l}\text { Mediu } \\
\mathrm{m}=00\end{array}$ & $\begin{array}{l}\text { Mediu } \\
\mathrm{m}=56\end{array}$ & $\begin{array}{l}\text { Mediu } \\
\mathrm{m}=38\end{array}$ & $\begin{array}{l}\text { Neutra } \\
l=35\end{array}$ & Medium $=00$ & Medium $=77$ & Medium $=54$ & $\begin{array}{l}\text { Neutra } \\
l=39\end{array}$ & $\begin{array}{l}\text { Mediu } \\
\mathrm{m}=00\end{array}$ & $\begin{array}{l}\text { Mediu } \\
\mathrm{m}=76\end{array}$ & $\begin{array}{l}\text { Mediu } \\
\mathrm{m}=50\end{array}$ \\
\hline & $\begin{array}{l}\text { Basic } \\
=71\end{array}$ & $\begin{array}{l}\text { High } \\
=00\end{array}$ & $\begin{array}{l}\text { High } \\
=23\end{array}$ & $\begin{array}{l}\text { High } \\
=27\end{array}$ & $\begin{array}{l}\text { Basic } \\
=80\end{array}$ & $\begin{array}{l}\text { High } \\
=00\end{array}$ & $\begin{array}{l}\text { High } \\
=20\end{array}$ & $\begin{array}{l}\text { High } \\
=21\end{array}$ & $\begin{array}{l}\text { Basic } \\
=27\end{array}$ & $\begin{array}{l}\text { High } \\
=00\end{array}$ & $\begin{array}{l}\text { High } \\
=00\end{array}$ & $\begin{array}{l}\text { High } \\
=24\end{array}$ & $\begin{array}{l}\text { Basic } \\
=24\end{array}$ & $\begin{array}{l}\text { High } \\
=00\end{array}$ & $\begin{array}{l}\text { High } \\
=02\end{array}$ & $\begin{array}{l}\text { High } \\
=24\end{array}$ \\
\hline NIV & & 1.00 & 2.28 & 2.66 & & 1.00 & 2.29 & 2.00 & & 1.00 & 1.99 & 2.31 & & 1.00 & 2.04 & 2.26 \\
\hline $\begin{array}{l}\text { Fertility } \\
\text { Rating }\end{array}$ & & Very Low & Adequate & High & & Very Low & Adequate & & & Very Low & & Adequate & & $\begin{array}{l}\text { Very } \\
\text { Low }\end{array}$ & & Adequatte \\
\hline
\end{tabular}

Table.2 Soil status for different coconut gardens (Continued)

MADHURE

SIRIYUR

\begin{tabular}{|c|c|c|c|c|c|c|c|c|c|c|c|c|c|c|c|c|}
\hline & \multicolumn{4}{|c|}{$0-30 \mathrm{~cm}$} & \multicolumn{4}{|c|}{$30-60 \mathrm{~cm}$} & \multicolumn{4}{|c|}{$0-30 \mathrm{~cm}$} & \multicolumn{4}{|c|}{$30-60 \mathrm{~cm}$} \\
\hline & pH & $\underset{(\mathrm{kg} / \mathrm{ha})}{\mathbf{N}}$ & $\begin{array}{c}\mathbf{P}_{2} \mathbf{O}_{5} \\
(\mathbf{k g} / \mathbf{h a})\end{array}$ & $\begin{array}{c}\mathrm{K}_{2} \mathrm{O} \\
(\mathrm{kg} / \mathrm{ha})\end{array}$ & pH & $\underset{(\mathrm{kg} / \mathrm{ha})}{\mathbf{N}}$ & $\begin{array}{c}\mathbf{P}_{2} \mathbf{O}_{5} \\
(\mathbf{k g} / \mathbf{h a})\end{array}$ & $\begin{array}{c}\mathrm{K}_{2} \mathrm{O} \\
(\mathrm{kg} / \mathrm{ha})\end{array}$ & pH & $\underset{(\mathrm{kg} / \mathrm{ha})}{\mathbf{N}}$ & $\begin{array}{c}\mathbf{P}_{2} \mathbf{O}_{5} \\
(\mathbf{k g} / \mathbf{h a})\end{array}$ & $\begin{array}{c}\mathrm{K}_{2} \mathrm{O} \\
(\mathrm{kg} / \mathrm{ha})\end{array}$ & pH & $\underset{(\mathbf{k g} / \mathbf{h a})}{\mathbf{N}}$ & $\begin{array}{c}\mathbf{P}_{2} \mathbf{O}_{5} \\
(\mathbf{k g} / \mathbf{h a})\end{array}$ & $\begin{array}{c}\mathrm{K}_{2} \mathrm{O} \\
(\mathrm{kg} / \mathrm{ha})\end{array}$ \\
\hline Maximum & 9.40 & 246.40 & 181.30 & 559.80 & 9.30 & 246.40 & 90.60 & 505.60 & 7.64 & 425.60 & 99.36 & 381.12 & 7.38 & 235.20 & 77.92 & 195.60 \\
\hline Minimum & 5.90 & 33.60 & 25.90 & 117.60 & 5.60 & 28.00 & 17.90 & 92.40 & 4.52 & 61.60 & 13.16 & 101.52 & 4.60 & 44.80 & 11.94 & 56.40 \\
\hline Average & 7.60 & 111.20 & 71.40 & 249.90 & 7.70 & 101.4 & 43.70 & 184.50 & 5.61 & 256.04 & 67.60 & 198.76 & 5.60 & 88.97 & 34.35 & 142.12 \\
\hline Standard deviation & 0.67 & 48.13 & 30.53 & 99.83 & 0.66 & 48.14 & 16.45 & 69.50 & 0.71 & 60.81 & 22.68 & 61.08 & 0.68 & 34.37 & 10.55 & 32.73 \\
\hline Standard error & 0.07 & 5.38 & 3.42 & 11.17 & 0.07 & 5.38 & 1.84 & 7.77 & 0.08 & 7.16 & 2.67 & 7.19 & 0.08 & 4.05 & 1.24 & 3.85 \\
\hline \multirow[t]{3}{*}{$\begin{array}{c}\text { Ratings } \\
\text { of samples }\end{array}$} & $\begin{array}{l}\text { Acidic } \\
=05\end{array}$ & $\begin{array}{l}\text { Low } \\
=80\end{array}$ & $\begin{array}{l}\text { Low } \\
=00\end{array}$ & $\begin{array}{l}\text { Low } \\
=06\end{array}$ & $\begin{array}{c}\text { Acidic } \\
=07\end{array}$ & $\begin{array}{l}\text { Low } \\
=80\end{array}$ & $\begin{array}{l}\text { Low } \\
=01\end{array}$ & $\begin{array}{l}\text { Low } \\
=20\end{array}$ & Acidic $=64$ & $\begin{array}{l}\text { Low } \\
=45\end{array}$ & $\begin{array}{l}\text { Low } \\
=02\end{array}$ & $\begin{array}{l}\text { Low } \\
=11\end{array}$ & $\begin{array}{c}\text { Acidic } \\
=65\end{array}$ & $\begin{array}{l}\text { Low } \\
=72\end{array}$ & $\begin{array}{l}\text { Low } \\
=08\end{array}$ & $\begin{array}{l}\text { Low } \\
=33\end{array}$ \\
\hline & Neutral $=27$ & Medium $=00$ & Medium $=27$ & Medium $=60$ & $\begin{array}{c}\text { Neutra } \\
l=26\end{array}$ & $\begin{array}{l}\text { Mediu } \\
\mathrm{m}=00\end{array}$ & $\begin{array}{c}\text { Mediu } \\
\mathrm{m}=58\end{array}$ & $\begin{array}{l}\text { Mediu } \\
\mathrm{m}=57\end{array}$ & $\begin{array}{c}\text { Neuta } \\
l=06\end{array}$ & Medium $=27$ & Medium $=17$ & Medium $=58$ & $\begin{array}{c}\text { Neutra } \\
l=7\end{array}$ & $\begin{array}{l}\text { Mediu } \\
\mathrm{m}=00\end{array}$ & $\begin{array}{l}\text { Mediu } \\
\mathrm{m}=61\end{array}$ & $\begin{array}{l}\text { Mediu } \\
\mathrm{m}=39\end{array}$ \\
\hline & $\begin{array}{l}\text { Basic } \\
=48\end{array}$ & $\begin{array}{l}\text { High } \\
=00\end{array}$ & $\begin{array}{l}\text { High } \\
=53\end{array}$ & $\begin{array}{l}\text { High } \\
=14\end{array}$ & $\begin{array}{c}\text { Basic } \\
=47\end{array}$ & $\begin{array}{l}\text { High } \\
=00\end{array}$ & $\begin{array}{l}\text { High } \\
=21\end{array}$ & $\begin{array}{l}\text { High } \\
=03\end{array}$ & $\begin{array}{l}\text { Basic } \\
=02\end{array}$ & $\begin{array}{l}\text { High } \\
=00\end{array}$ & $\begin{array}{l}\text { High } \\
=53\end{array}$ & $\begin{array}{l}\text { High } \\
=03\end{array}$ & $\begin{array}{c}\text { Basic } \\
=00\end{array}$ & $\begin{array}{l}\text { High } \\
=00\end{array}$ & $\begin{array}{l}\text { High } \\
=03\end{array}$ & $\begin{array}{l}\text { High } \\
=00\end{array}$ \\
\hline \multirow[t]{2}{*}{ NIV } & & 1.00 & 2.66 & 2.10 & & 1.00 & 2.25 & 1.79 & & 1.38 & 2.71 & 1.85 & & 1.00 & 1.83 & 1.54 \\
\hline & & Very Low & High & Adequate & & Very Low & & & & & Very high & & & $\begin{array}{l}\text { Very } \\
\text { Low }\end{array}$ & & \\
\hline
\end{tabular}


Table.3 Fertilizer requirement $(\mathrm{kg})$ for the study area depending on status of nutrient on a grid basis

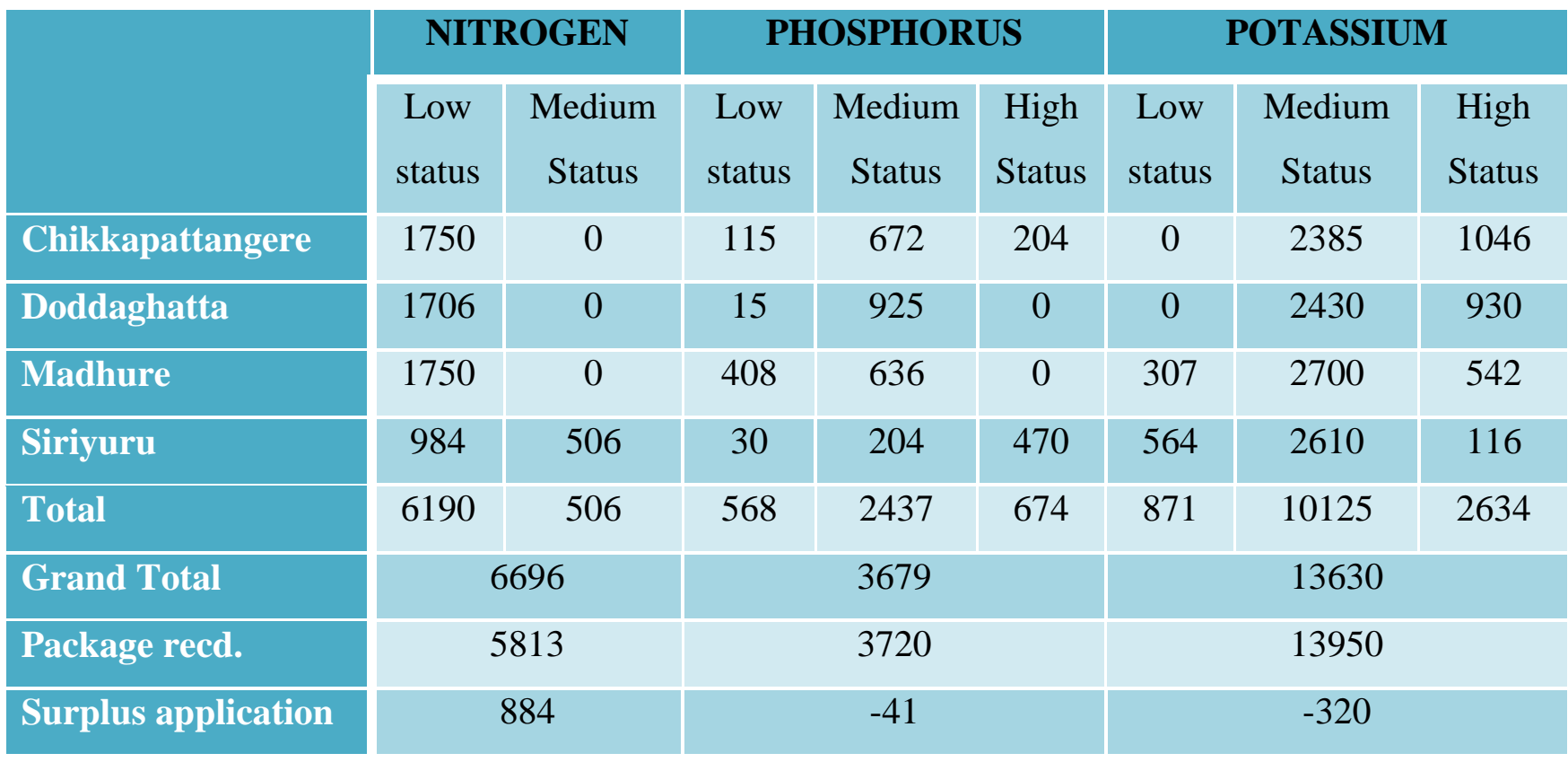

Fig.1 Study location in selected districts of Karnataka in India
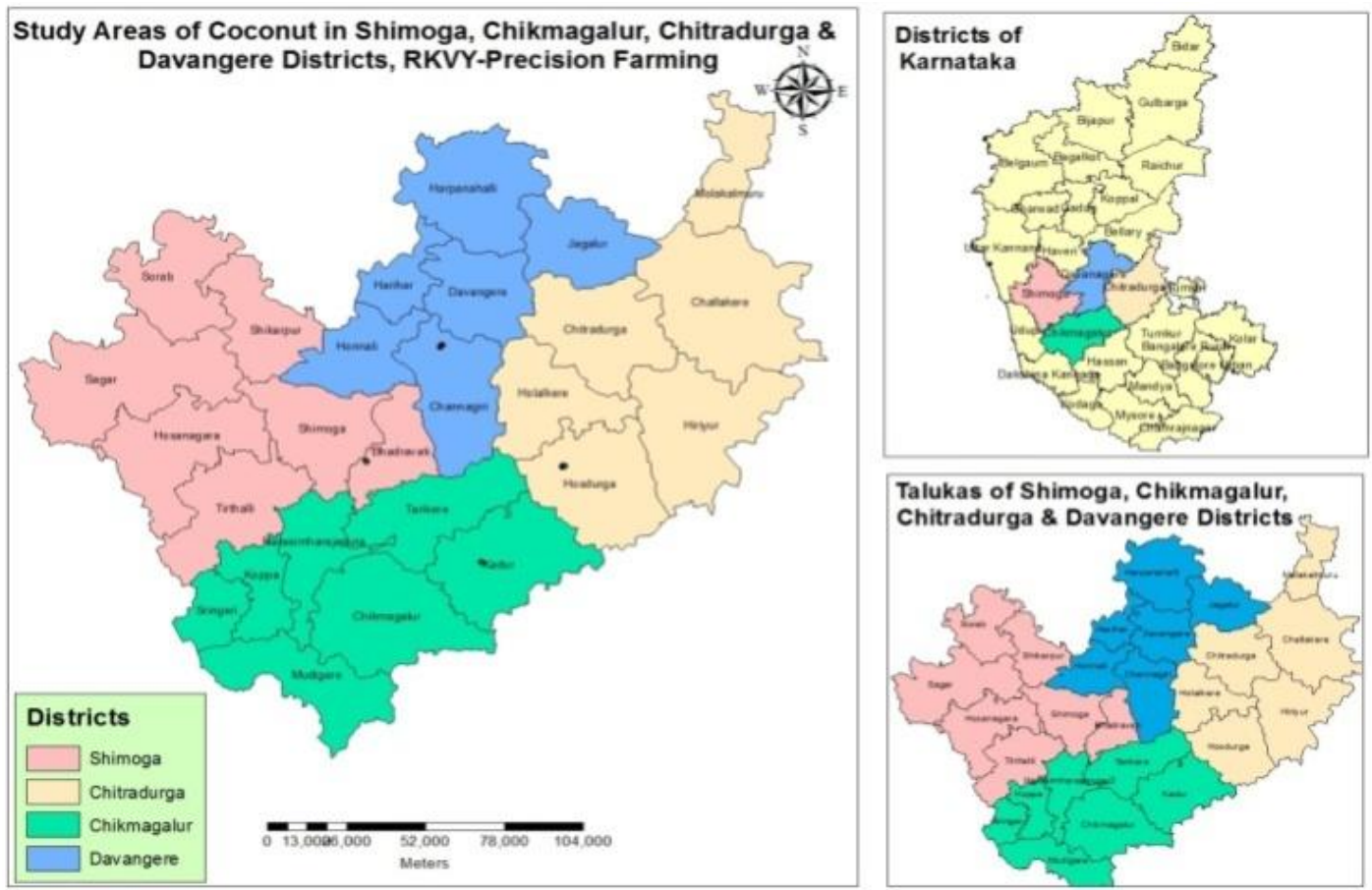

Fig.2 (a) Soil variability map of major nutrients for Chikkapattanagere 


\section{CHIKKAPATTANAGERE}

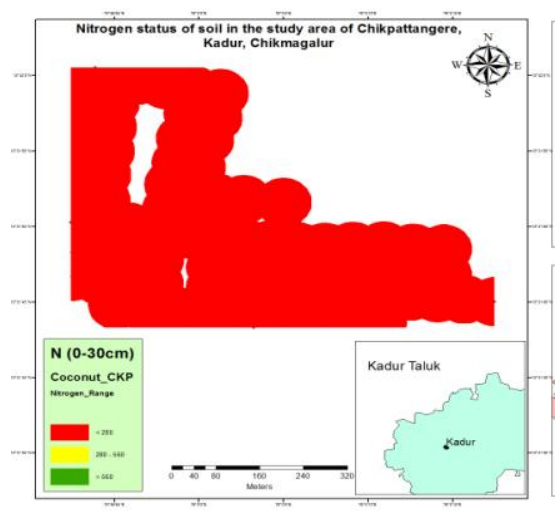

Nitrogen $(0-30 \mathrm{~cm})$

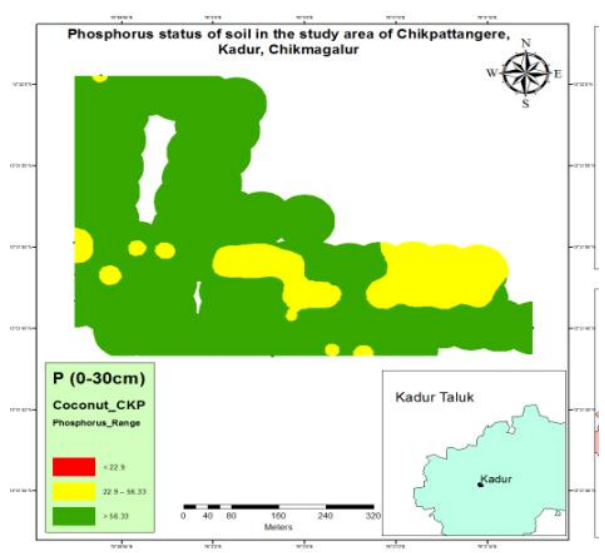

Phosphorus (0-30 cm)

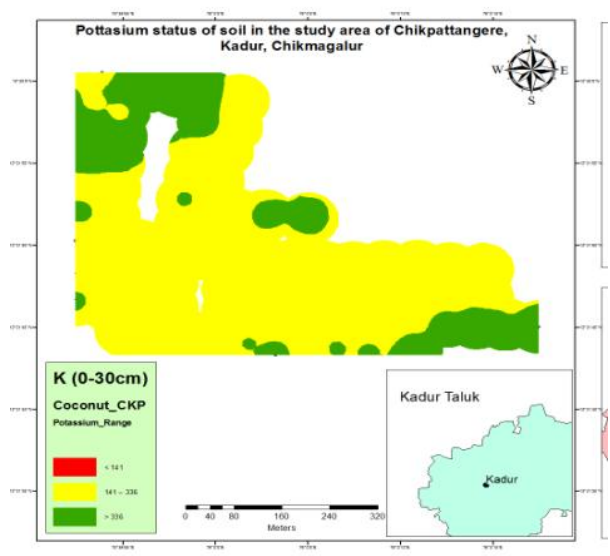

Potassium (0-30 cm)

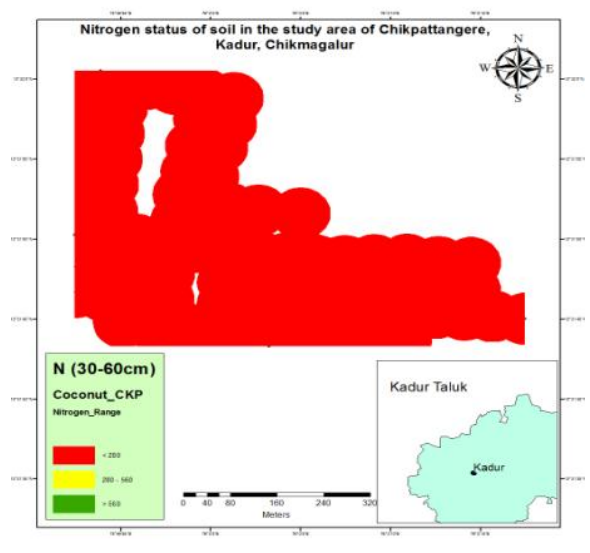

Nitrogen $(30-60 \mathrm{~cm})$

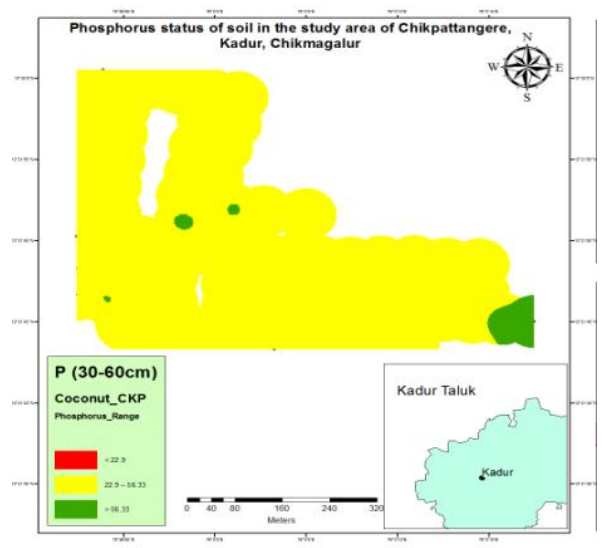

Phosphorus (30-60 cm)

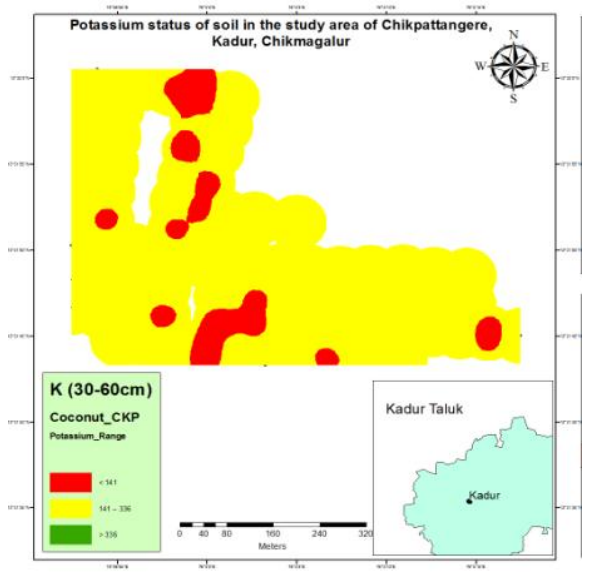

Potassium $(0-30 \mathrm{~cm})$

Fig.2 (b) Soil variability map of major nutrients for Doddaghatta 


\section{DODDAGHATTA}

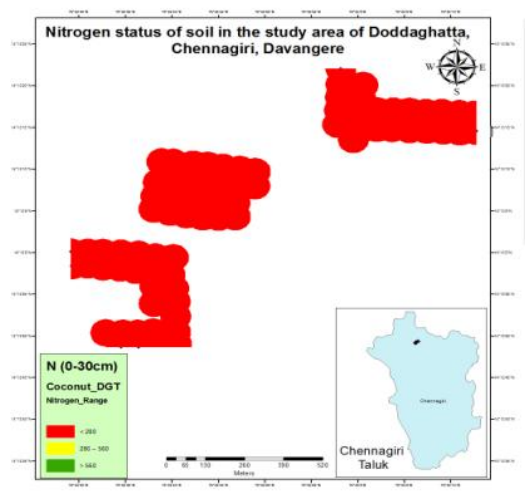

Nitrogen $(0-30 \mathrm{~cm})$

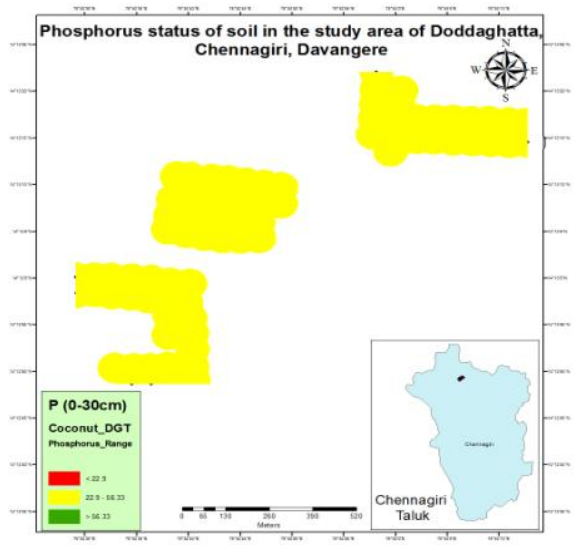

Phosphorus $(0-30 \mathrm{~cm})$

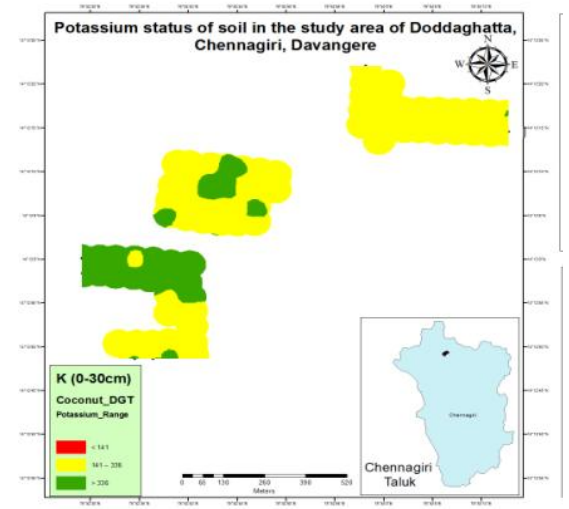

Potassium $(0-30 \mathrm{~cm})$

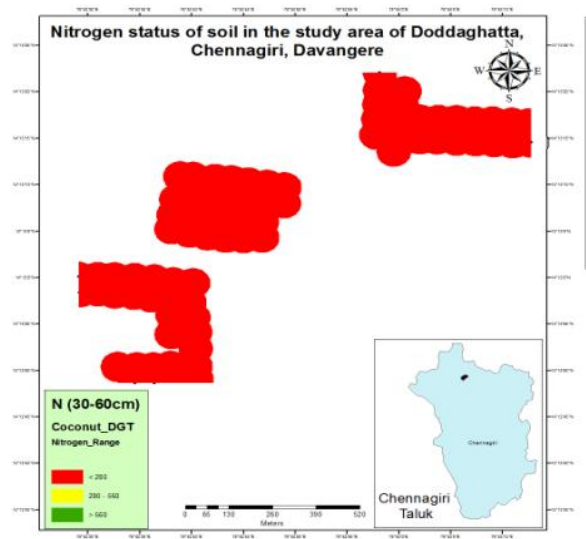

Nitrogen $(30-60 \mathrm{~cm})$

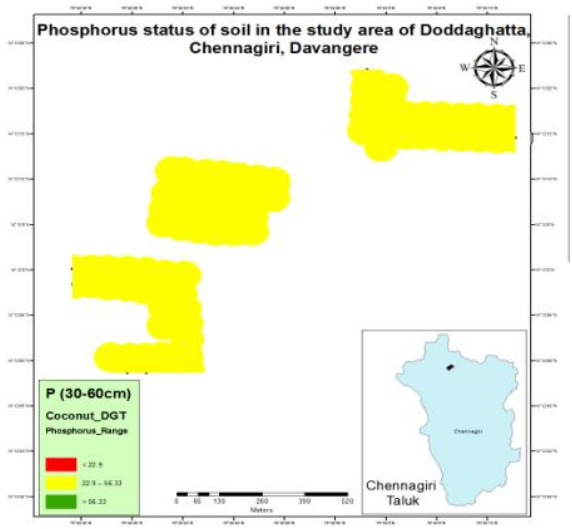

Phosphorus $(30-60 \mathrm{~cm})$

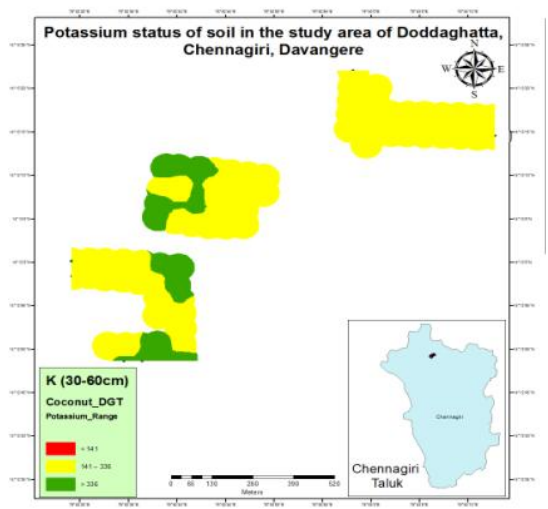

Potassium $(0-30 \mathrm{~cm})$

Fig.2 (c) Soil variability map of major nutrients for Siriyur 


\section{SIRIYUR}

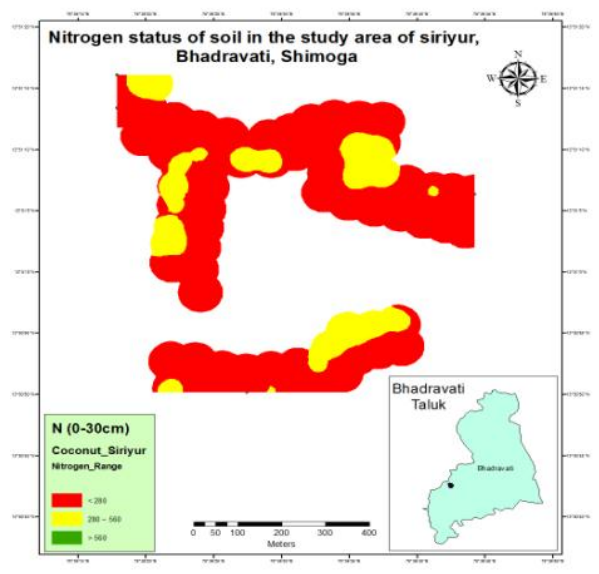

Nitrogen $(0-30 \mathrm{~cm})$

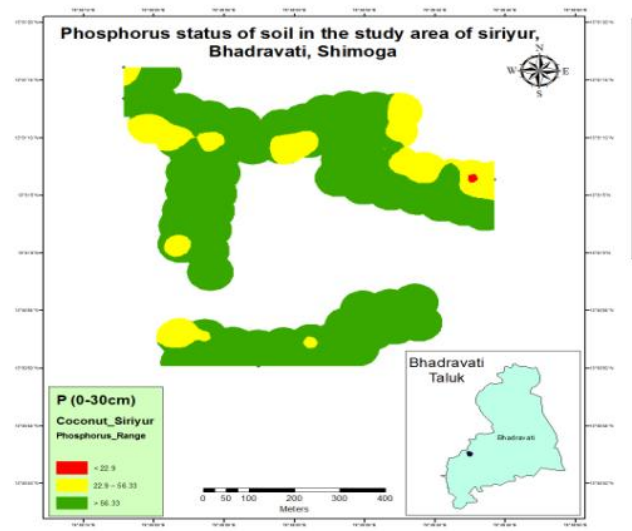

Phosphorus $(0-30 \mathrm{~cm})$

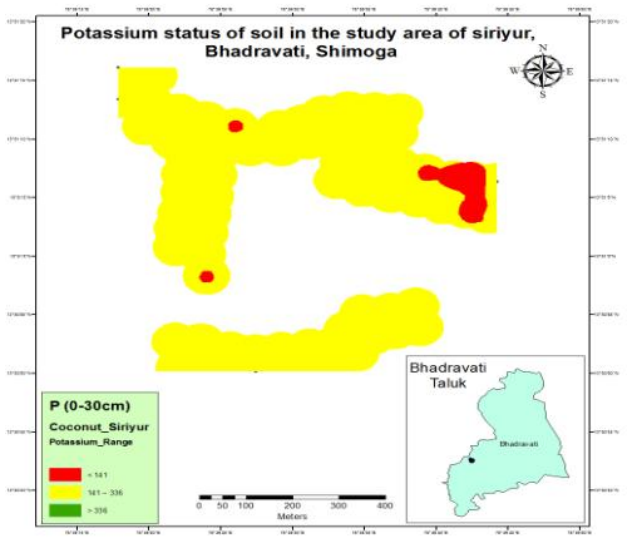

Potassium $(0-30 \mathrm{~cm})$

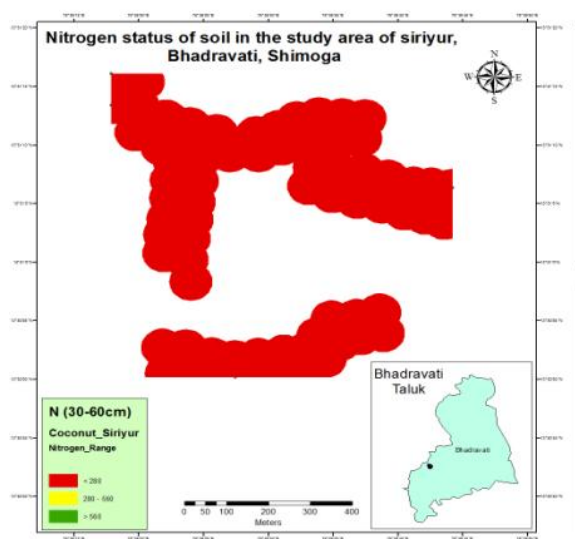

Nitrogen $(30-60 \mathrm{~cm})$

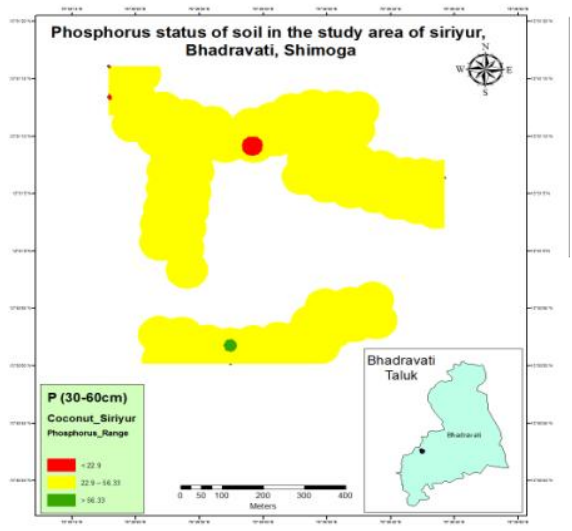

Phosphorus (30-60 cm)

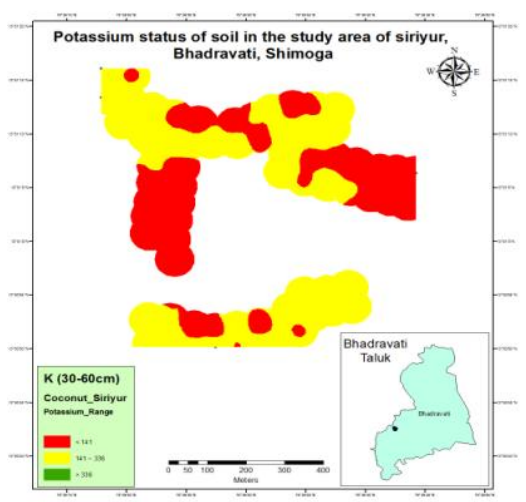

Potassium $(0-30 \mathrm{~cm})$

Fig.2 (d) Soil variability map of major nutrients for Madhure 


\section{MADHURE}

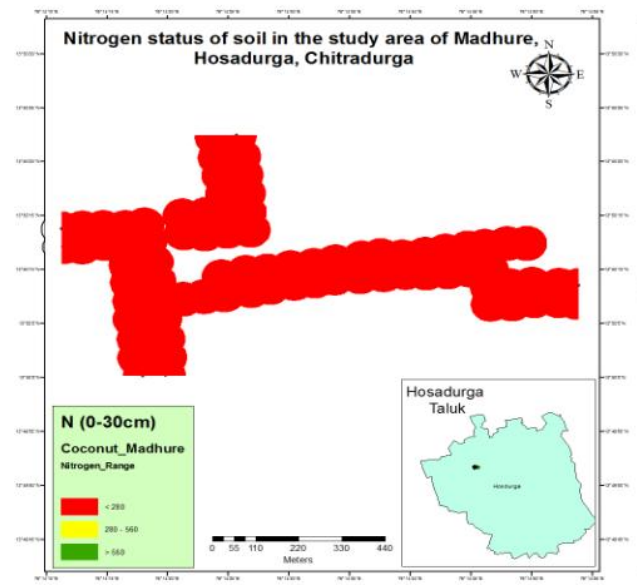

Nitrogen $(0-30 \mathrm{~cm})$

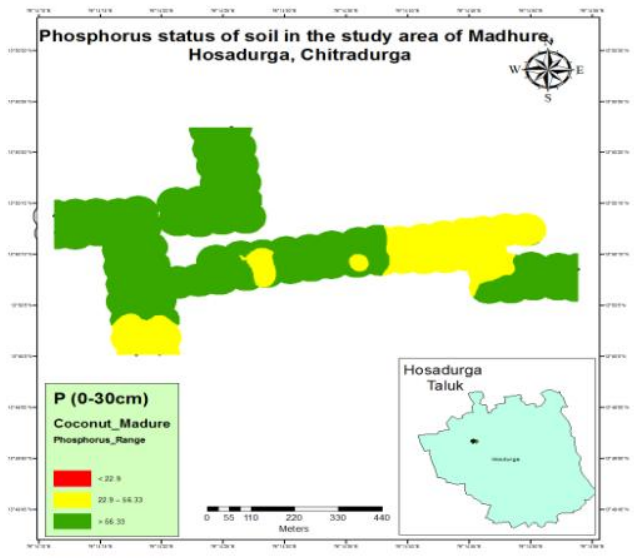

Phosphorus $(0-30 \mathrm{~cm})$

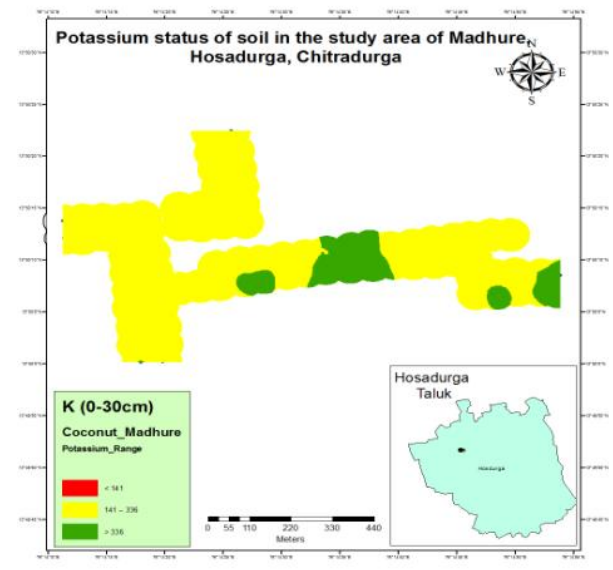

Potassium $(0-30 \mathrm{~cm})$

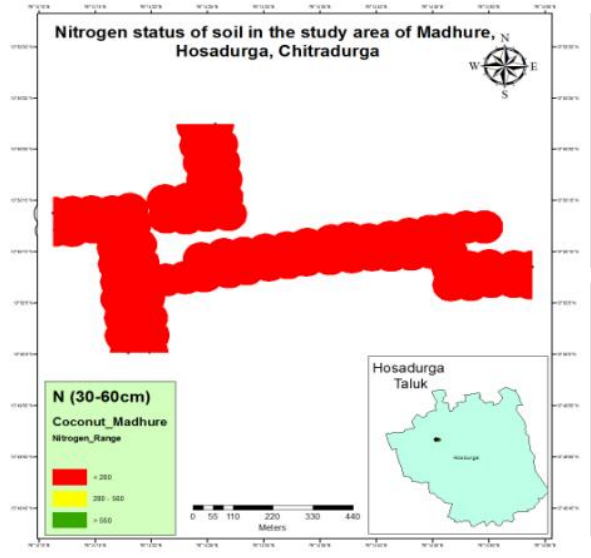

Nitrogen $(30-60 \mathrm{~cm})$

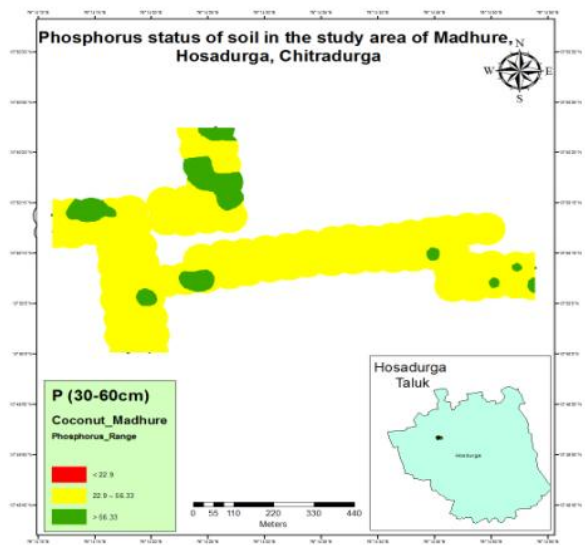

Phosphorus $(30-60 \mathrm{~cm})$

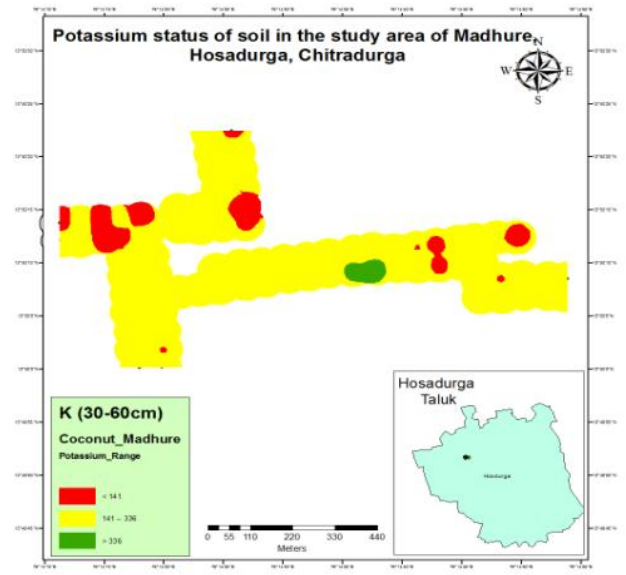

Potassium $(0-30 \mathrm{~cm})$

Fig.3 Quantity of fertilizer required in traditional and site specific recommendations for coconut 


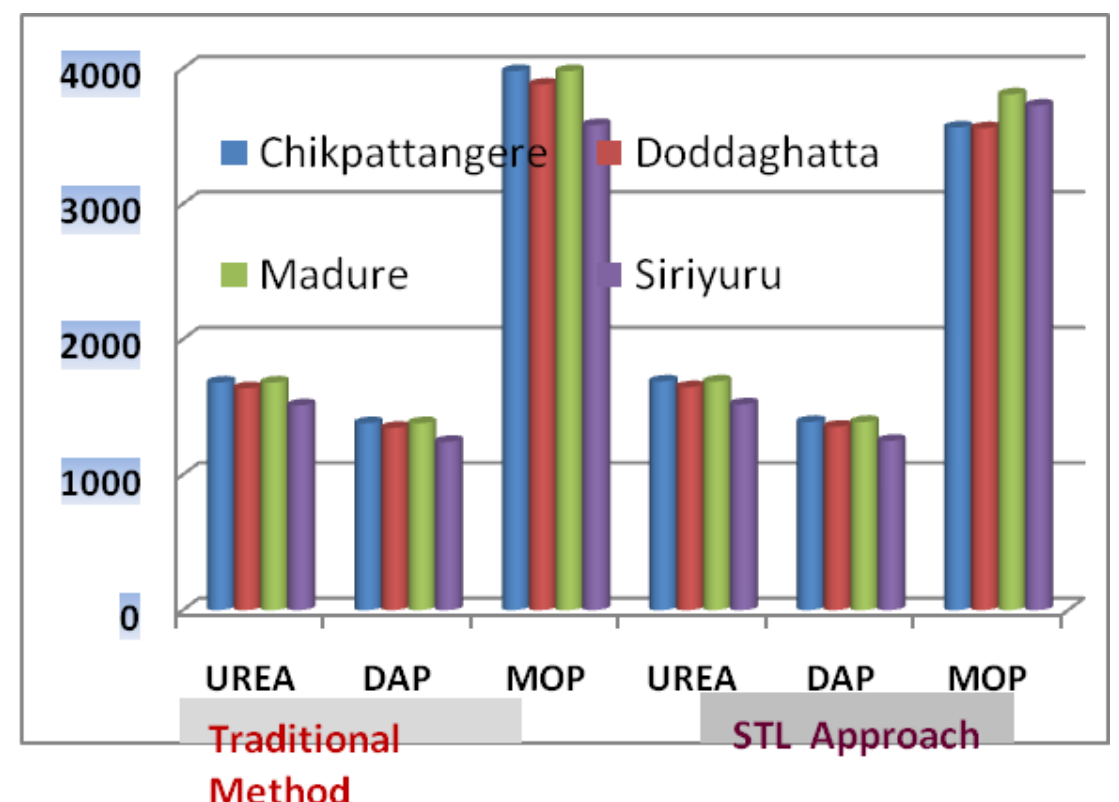

Based on these variations of major nutrient contents on a grid basis, a base map of the study area is considered for further classification into fertility status maps and same has been drawn using GIS technology. A soil variability map for major nutrients in the selected study area is presented in figure 2(a) to 2(d). Across locations, for each grid, a chart is prepared concerning different macro nutrients for low, medium and high status.

The serious concern in perennial performance was mainly due to the location to location fertility status, sustained nutrient requirement and low nutrient use efficiency (Bhat and Sujatha, 2009). Predominantly nitrogen across all locations found to be low due to inherent low content, faster degradation owing to a higher temperature, increased use efficiency of element under assured supply of irrigation are the reasons or low content apart from abiotic stresses acting (Barman et al., 2013). Phosphorus requirement in grown up plants is not in higher proportion and variations observed across locations are mainly attributed to supply from various sources and its retention in soil thereby increases reserved source due to active fixation (Mahesh Kumar et al., 2015). Potassium variations mainly attributed to nativity of soil status and supply from different sources (Dhanashekaran Pandian and Mohamed Haroon, 2014). The nature and amount of variation exists for different nutrient largely depends on management factor, supply pattern from different sources and well supported in the studies of Sabita Soman and Byju (2013).

Fertility status maps envisage that nitrogen status remained low while that of phosphorus sharing almost medium and high in equal proportion (Bopathi and Sharma, 2006) while 68 per cent high concerning potash for top soil depth of $0-30 \mathrm{~cm}$. Hence it becomes crucial to managing these elements (Mahesh Kumar et al., 2015). Here based on package recommendations, soil test value approach is employed to make further calculations grid wise (Table 3). The total requirement of fertilizers based on package recommendation for these areas is indicated in Table 3 along with calculated STV approach. It is observed from the above result that coconut garden soils across all locations are to be supplied with slightly higher nitrogen $(884 \mathrm{~kg})$ and lower potassium $(320 \mathrm{~kg}$ ) with phosphorus (41 kg) thereby given re-allocation of these resources based on site specificity. Usually, 
farmers apply in the form of DAP, urea and muriate of potash. The total requirement of fertilizers based on package recommendation for these areas is indicated in pictorial depiction (Fig. 3) respectively along with calculated soil test value approach.

The data permit to infer that across all locations nitrogen status remained low and hence achieved lowest fertility rating. Fertility ratings for phosphorus and potassium varied from high to adequate as samples analyzed shared more of medium to high status. Hence, it is observed from the above result that for coconut crop, across all locations, slightly nitrogen application needs to be applied more while potassium needs to be applied less with almost no perceptible change in the application of phosphorus.

\section{Acknowledgments}

The authors are thankful to the State Agriculture Department, Karnataka, India for providing financial assistance and the University of Agricultural and Horticultural Sciences, Shivamogga during this research work. We are thankful to farmers and agricultural officers for village level supervision.

\section{References}

Anonymous, 2016. Area, production and productivity of arecanut.Indiastat.com

Barman, D., Saho, R. N., Kalra, N., Kamble, K. and Kundu, D.K. 2013. Homogenous soil fertility mapping through GIS for site specific nutrient management by QUEFTS model. Indian Journal of Soil Conservation. 41(3): 257-261.

Bhat, R. and Sujatha, S. 2009. Soil fertility and nutrient uptake by arecanut as affected by level and frequency of fertigation in a laterite soil. Agriculture Water Management 96(3): 445-456.
Dhanashekaran Pandiyan, R. and Mohamed Harron, A. R. 2014. Soil nutrient status mapping through GIS technique of direct seeding rice cultivating area of Ramnad district. Trends in Bioscience. 7(2): 3722-3726.

Hartemink, A.E. 2005. Nutrient stocks, nutrient cycling and soil change in cocoa ecosystem: A review. Advances in Agronomy. 86: 227-253.

Jackson, M.L. 1973. Soil Chemical Analysis, prentice Hall of India Private Limited, New Delhi.

Kabata-Pendias, A. 2004. Soil plant transfer of trace elements-an environmental issue. Geoderma. 122: 143-149.

Mahesh Kumar, Panwar, N.R., Pandey, C.R. and Jatav, M.K 2015. Spatial distribution of available nutrients in the potato growing areas of Banaskantha district of Gujarat. Potato Journal. 42(2): 130-136.

Muhr, C.R., Datta, N.P., Sankrasbramoney, H., Lesley, V.K. and Donahue, R.L. 1965. Soil testing in India. USADI, New Delhi, India.

Olsen, S.R., Cole, C.V., Watanate, F.S. and Dean, A.L. 1954. Estimation of available phosphorus in soil by extraction with sodium bi carbonate. USDA Circ. 939.

Ramamoorthy, B. and Bajaj, J.C. 1969. Available N, P and $\mathrm{K}$ status of Indian soils. Fertilizer News. 14: 24-26.

Sabitha Soman and Byju, G. 2013. Geospatial assessment and mapping of selected soil physical and chemical properties at Farm Level: A case study in an Ultisol. Journal of Root Crops. 39(2): 73-86.

Shetty, Y.V., Nagamma, M.S., Dinesh Kumar, M. and Jayaprakash, S. 2008. Fertility status in arecanut garden soils of Kanataka. Karnataka Journal of Agricultural Sciences. 21(4): 503-506.

Subbaiah, B.V. and Asija, G.L. 1956. A rapid procedure for estimation of available 
nitrogen in soils. Current Science. 25: 259-260.

Tandon, H. L. S. 1993. Methods of analysis of soils, plants, water and fertilizers and consultation organization. New Delhi, India: 31

Trehan, S.P., Upadhyaya, N.C. Sud, K.C., Kumar, M., Jatav, M.K. and Lal, S.S. 2008. Nutrient Management in Potato,
CPRI, Shimla, India, Technical bulletin: 90.

Vijaya Kumar, M., Bakiyathu Saliha, B., Kannan, P. and Mahendran, P.P. 2015. Delineation and GIS mapping of soil nutrient status of sugarcane growing tracts of Theni district, Tamil Nadu. African Journal Agricultural Research. 10(3): 3281-3291.

\section{How to cite this article:}

Dinesh Kumar, M., Binny Gopal and Dhananjaya, B.C. 2018. GIS Based Site Specific Major Nutrient Maps and Recommendations for Coconut (Cocus nucifera L.) Gardens. Int.J.Curr.Microbiol.App.Sci. 7(05): 3643-3654. doi: https://doi.org/10.20546/ijcmas.2018.705.420 